\title{
The Relationship between Prostate Inflammation and Lower Urinary Tract Symptoms: Examination of Baseline Data from the REDUCE Trial
}

\author{
J. Curtis Nickela, ${ }^{*}$, Claus G. Roehrborn $^{\mathrm{b}}$, Michael P. O'Learyc, David G. Bostwick ${ }^{\mathrm{d}}$, Matthew \\ C. Somerville ${ }^{\mathrm{e}}$, and Roger S. Rittmaster ${ }^{\mathrm{f}^{\prime}}$ \\ aDepartment of Urology, Queen's University, Kingston General Hospital, Kingston, ON, Canada \\ bDepartment of Urology, The University of Texas Southwestern Medical Center at Dallas, Dallas, TX, USA \\ cDepartment of Surgery, Harvard Medical School, Division of Urology, Brigham and Women's Hospital, \\ Boston, MA, USA
}

dBostwick Laboratories, Glen Allen, VA, USA

eGlaxoSmithKline, Biostatistics, Research Triangle Park, NC, USA

fGlaxoSmithKline, Urology Clinical Development and Medical Affairs, Research Triangle Park, NC, USA

\begin{abstract}
Objective-The ongoing REDUCE trial is a 4-yr, phase 3, placebo-controlled study to determine if daily dutasteride $0.5 \mathrm{mg}$ reduces the risk of biopsy detectable prostate cancer. Prostate biopsies performed in all men prior to entry were centrally reviewed, thus allowing an examination of the relationship between inflammatory changes and lower urinary tract symptoms (LUTS).
\end{abstract}

Methods-Eligible men were aged 50-75 yr, with serum prostate-specific antigen $\geq 2.5 \mathrm{ng} / \mathrm{ml}$ and $\leq 10 \mathrm{ng} / \mathrm{ml}(50-60 \mathrm{yr})$, or $\geq 3.0 \mathrm{ng} / \mathrm{ml}$ and $\leq 10 \mathrm{ng} / \mathrm{ml}$ ( $>60 \mathrm{yr}$ ) and an International Prostate Symptom Score (IPSS) $<25$ (or $<20$ if already on alpha-blocker therapy). Acute prostatitis was an exclusion criterion. For a given individual, inflammation was assessed across all cores and the amount of inflammation scored as none (0), mild (1), moderate (2), or marked (3). LUTS was assessed with the use of the IPSS. The relationship between inflammation scores (averaged over all cores) and total IPSS; grouped IPSS $(0-3,4-7,8-11,12-15,16-19, \geq 20)$; and irritative, obstructive, and nocturia subscores was determined by Spearman rank correlations. The relative contribution of inflammation, age, and body mass index was then examined with the use of linear regression analyses.

Results-Data were available for 8224 men. Statistically significant but relatively weak correlations were found between average and maximum chronic inflammation and IPSS variables (correlation coefficients, 0.057 and 0.036 , respectively; $p<0.001$ for total IPSS). Both age and

*Corresponding author. J. Curtis Nickel, Professor of Urology, Kingston General Hospital, Kingston, Ontario, Canada K7L 2V7. Tel. 613-548-2497; Fax: 613-545-1970. E-mail address: jcn@queensu.ca (J.C. Nickel).

Publisher's Disclaimer: This is a PDF file of an unedited manuscript that has been accepted for publication. As a service to our customers we are providing this early version of the manuscript. The manuscript will undergo copyediting, typesetting, and review of the resulting proof before it is published in its final citable form. Please note that during the production process errors may be discovered which could affect the content, and all legal disclaimers that apply to the journal pertain.

Presented at the 22nd Annual EAU Congress, Berlin, Germany, March, 2007

Take-home message

Central review of initial prostate biopsies performed in men enrolled into the REDUCE trial allowed examination of the relationship between inflammation and lower urinary tract symptoms (LUTS). In this population, there was a relationship between LUTS and chronic inflammation. 
average chronic inflammation were significant in the linear regression after adjustment for other covariates; for both variables, more severe inflammation was associated with higher IPSS scores.

Conclusions-In the REDUCE population, there is evidence of a relationship between the degree of LUTS and the degree of chronic inflammation. Study entry criteria that selected older men and decreased enrolment of men with a greater degree of inflammation and LUTS may have limited the strength of this relationship. The impact of baseline prostate inflammation on progression of LUTS and/or associated complications will be determined during 4-yr longitudinal follow-up.

\section{Introduction}

It was suggested a number of years ago that prostate inflammation may be the third component (the first two being dihydrotestosterone-mediated "static" prostate enlargement and "dynamic" alpha-receptor-mediated muscle tension) in determining the association between benign prostatic hyperplasia (BPH) and lower urinary tract symptoms (LUTS) [1]. There has been a renewed interest in examining the role of histological inflammation in the pathogenesis and progression of BPH. Evolving basic science data suggest that asymptomatic prostatic inflammation is associated with the development of histological BPH [2,3]. Inflammation detected in prostate biopsies performed at baseline assessment in a subgroup of over 1000 patients enrolled in the Medical Therapies of Prostate Symptoms (MTOPS) study predicted progression events such as symptom worsening, acute urinary retention, and need for surgery in placebo-treated patients [4].

REDUCE (REduction by DUtasteride of prostate Cancer Events) is an ongoing, large-scale, 4-yr clinical trial designed to determine if and to what extent the dual $5 \alpha$-reductase inhibitor dutasteride reduces the risk of biopsy detectable prostate cancer compared with placebo in men at increased risk of developing prostate cancer [5]. The entrance criteria for REDUCE included the requirement of a prostate cancer-negative biopsy prior to enrolment. The data from the entrance biopsy have enabled additional protocol-defined investigations to be made, including examination of the baseline relationships between histological prostate inflammation and LUTS (measured with the International Prostate Symptom Score [IPSS]). This report examines the association between LUTS and histological prostate inflammation in the REDUCE population.

\section{Materials and methods}

\subsection{Study conduct}

REDUCE is a 4-yr, randomised, double-blind, placebo-controlled study evaluating the efficacy and safety of once-daily $0.5-\mathrm{mg}$ dutasteride in reducing the risk of biopsy detectable prostate cancer in men at increased risk of developing prostate cancer [4]. Entry criteria for REDUCE included men aged 50-75 $\mathrm{yr}$ with a serum prostate-specific antigen (PSA) $\geq 2.5 \mathrm{ng} / \mathrm{ml}$ and $\leq 10$ $\mathrm{ng} / \mathrm{ml}(50-60 \mathrm{yr})$, or $\geq 3.0 \mathrm{ng} / \mathrm{ml}$ and $\leq 10 \mathrm{ng} / \mathrm{ml}$ ( $>60 \mathrm{yr}$ ) and a negative prostate biopsy within 6 mo prior to enrolment. Key exclusion criteria included a prostate volume $>80 \mathrm{ml}$, an IPSS $\geq 25$ (or $\geq 20$ if already on alpha-blocker therapy for BPH), concurrent and/or previous use within the past 12 mo of a $5 \alpha$-reductase inhibitor, and an investigator-determined diagnosis of acute prostatitis or acute bacterial prostatitis within 6 mo of study entry.

\subsection{Study assessments}

The IPSS was used to assess LUTS. The first seven items quantitatively measure irritative (frequency, urgency, and nocturia) and obstructive symptoms (incomplete emptying, intermittency, weak stream, and straining); each question is scored from $0-5$, giving a maximum score of 35 [6]. A supplemental item (Q8) assesses bother on a scale of 0-6. A central pathology laboratory (Bostwick Laboratories, Richmond, VA, USA) graded average acute and 
chronic inflammation on a 4-point scale (none [0], mild [1], moderate [2], or severe [3]) on the basis of average cell density and extent of tissue involvement in each biopsy core (Fig. 1). Chronic inflammation consisted chiefly of lymphocytes with a variable number of plasma cells, macrophages, and, rarely, eosinophils. Acute inflammation consisted of neutrophils. Mild inflammation was defined as small scattered or patchy aggregates and the presence of nests of inflammatory cells, usually no more than $10-15$ cells per nest. Moderate inflammation was characterised by larger aggregates and nests, usually more than about 15 cells, invariably multifocal. Moderate inflammation was noticeable at low magnification. Sheets of inflammatory cells or extensive multifocal confluent masses of cells were noted in severe inflammation, which was obvious and noticeable at any magnification. Additional criterion for severe acute inflammation was tissue destruction of any size.

\subsection{Data analysis}

Baseline demographic variables and IPSS were summarised for men with inflammation (grade 1,2 , or 3 ) versus without (grade 0 ). Wilcoxon rank sum test was used to determine differences by presence versus absence of maximum chronic inflammation for total IPSS; grouped IPSS $(0-3,4-7,8-11,12-15,16-19, \geq 20)$; and irritative, obstructive, and nocturia sub-scores. Spearman rank correlations (range between -1 and +1 ) determined the relationship between inflammation scores (averaged overall cores) and the various IPSS measures. Acute and chronic inflammation were analysed independently because each subject had a chronic inflammation value and an acute inflammation value. $p$ values for correlations were interpreted with the use of the Bonferroni approach to adjust for multiple comparisons ( 5 different IPSS measures for both acute and chronic inflammation resulted in 10 comparisons, hence the $p$ value cut off $=0.05 / 10=0.005$ ). Linear regression was used to examine the relative contribution of inflammation, age, and body mass index (BMI) to BPH symptoms. All analyses were performed with the use of SAS ${ }^{\circledR}$, version 8.2 (SAS Institute, Cary, NC, USA).

\section{Results}

\subsection{Baseline characteristics}

Baseline characteristics are shown in Table 1. Data were available for 8224 men (median, 9 cores per subject). At baseline $15.4 \%$ had acute inflammation, $77.6 \%$ had chronic inflammation, and $21.6 \%$ had no inflammation. For those men with acute inflammation, $97.9 \%$ had mild, $1.9 \%$ had moderate, and $0.2 \%$ had severe inflammation. For those with chronic inflammation, the values were $89.0 \%, 10.7 \%$, and $0.3 \%$, respectively. Minor differences in age, serum PSA, and prostate volume were observed between men with and without histological inflammation

\subsection{Histological inflammation and the IPSS}

Total IPSS score and subscores were higher in the group of patients with histological chronic inflammation at baseline compared with those with no chronic inflammation. The differences were small but statistically significant (Table 1). . Statistically significant correlations were found between average chronic inflammation score and the IPSS variables (Table 2). However, the magnitude of these correlations was small, indicating very weak associations. No statistically significant associations were found between average acute inflammation and any IPSS variable examined (Table 2). Correlations between both acute and chronic inflammation and the IPSS variables examined were also performed with the use of maximum inflammation score (maximum of all cores). Correlation between maximum acute and chronic inflammation, and IPSS and subscores are presented in Table 3).

In the regression analysis that included age, BMI, and average acute and chronic inflammation, higher values of age and average chronic inflammation were significantly associated with 
higher IPSS scores ( $p<0.0001$ and $p=0.0005$, respectively). The contributions of BMI and average acute inflammation to the relationship were not statistically significant.

\section{Discussion}

REDUCE has provided the largest body of data (over 8000 men) to date, which examine relationships between histological prostate inflammation and prostate-related symptoms. An initial evaluation of a subset of the REDUCE population (5597 men), who completed a validated prostatitis questionnaire, failed to demonstrate a clinically meaningful association between baseline prostate inflammation and prostatitis-like symptoms [7]. In that study, the aim was to correlate histological inflammation and prostatitis-like symptoms (with or without LUTS) defined by predetermined prostatitis pain scores. The present study examines the larger number of men who completed the IPSS, and the aim was to correlate histological inflammation with BPH-related LUTS. Given that the mean prostate volume in this cohort was $46 \mathrm{ml}$ and the PSA $5.9 \mathrm{ng} / \mathrm{ml}$, and that the prostate biopsy did not show prostate cancer, it could be predicted that the REDUCE population would be enriched with men with BPH. Chronic histological inflammation was found in more than $77 \%$ of men in REDUCE, a prevalence reflecting a $\mathrm{BPH}$ population of ageing men [8].

The presented baseline data suggest a weak relationship between the degree of chronic inflammation and LUTS at baseline in the REDUCE population. The weakness of this relationship may be due to study entry criteria that selected older men and excluded men with clinical prostatitis or severe LUTS. No association was found between the degree of acute inflammation and LUTS at baseline in the REDUCE population. The number of patients enrolled with acute inflammation was low (15.4\%), and of these the majority (97.9\%) had mild inflammation. Acute prostatitis or acute bacterial prostatitis within 6 mo of study entry was an exclusion criterion in this study.

What could be the value of diagnosing prostate inflammation, either in a biopsy to rule out prostate cancer or by some yet to be discovered prostate-specific inflammation biomarker? Given the ubiquitous nature of chronic prostate inflammation in ageing men, and the weak correlation found in this study between chronic inflammation and LUTS, the clinical value of diagnosing prostate inflammation in men with LUTS may appear limited. The pattern of inflammation in prostate tissue is not homogeneous; inflammation noted is usually mild and acute, and chronic prostatitis can coexist in the same individual. However, there is an increasing understanding of the important role that prostate inflammation may have in promoting development of histological BPH (and perhaps prostate cancer) [2,3]. Furthermore, an examination of baseline prostate biopsies in a subgroup of 1197 patients in the Medical Therapies of Prostate Symptoms (MTOPS) study found that men in the placebo arm with inflammation were significantly more likely to experience symptom worsening or acute urinary retention than those without inflammation (5.6\% vs. $0.0 \%, p=0.003)$ [4], although-similar to the present findings - there were only weak correlations at baseline between inflammation and signs, symptoms, and markers of LUTS and clinical BPH. The absence of a clinically meaningful relationship between the parameters, however, does not preclude the possibility that such parameters may be strongly correlated with changes over time. The findings from MTOPS indeed suggest that inflammation is a predictor or even a driver of BPH progression. Longitudinal 4-yr follow-up in the REDUCE study may therefore provide further insight into the impact of baseline prostate inflammation on progression of LUTS and/or associated complications. 


\section{Conclusion}

In the REDUCE population, there is evidence of a weak relationship between the degree of LUTS and the degree of chronic inflammation. The impact of baseline prostate inflammation on progression of LUTS and/or associated complications will be determined during 4-yr longitudinal follow-up.

\section{References}

1. Nickel JC. Prostatic inflammation in benign prostatic hyperplasia[-] the third component? Can J Urol 1994;1:1-4. [PubMed: 12834558]

2. Kramer G, Mitteregger D, Marberger M. Is benign prostatic hyperplasia (BPH) an immune inflammatory disease? Eur Urol 2007;51:1202-1216. [PubMed: 17182170]

3. Sciarra A, Di Silverio F, Salciccia S, Autran Gomez AM, Gentilucci A, Gentile V. Inflammation and chronic prostatic diseases: evidence for a link? Eur Urol 2007;52:964-972. [PubMed: 17618043]

4. Roehrborn CG. Definition of at-risk patients: baseline variables. BJU Int 2006;97:7-11. [PubMed: 16507046]

5. Andriole G, Bostwick D, Brawley O, et al. Chemoprevention of prostate cancer in men at high risk: rationale and design of the reduction by dutasteride of prostate cancer events (REDUCE) trial. J Urol 2004;172:1314-1317. [PubMed: 15371831]

6. Barry MJ, Fowler FJ Jr, O'Leary MP, et al. The American Urological Association symptom index for benign prostatic hyperplasia. The Measurement Committee of the American Urological Association. J Urol 1992;148:1549-1557. [PubMed: 1279218]discussion 64

7. Nickel JC, Roehrborn CG, O'Leary MP, Bostwick DG, Somerville MC, Rittmaster RS. Examination of the relationship between symptoms of prostatitis and histologic inflammation: baseline data from the REDUCE chemoprevention trial. J Urol. In press

8. Nickel JC, Downey J, Young I, Boag S. Asymptomatic inflammation and/or infection in benign prostatic hyperplasia. BJU Int 1999;84:976-981. [PubMed: 10571623] 


\section{Mild and Moderate Acute and Chronic Inflammation}

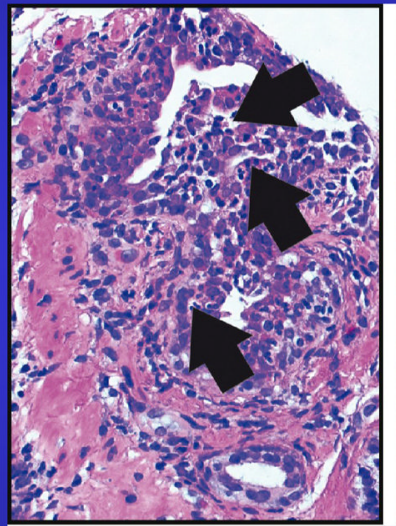

Mild acute inflammation

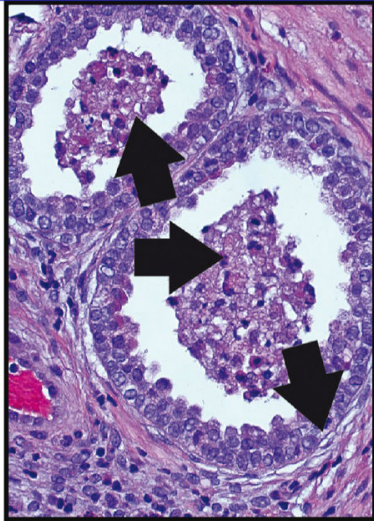

Moderate acute inflammation

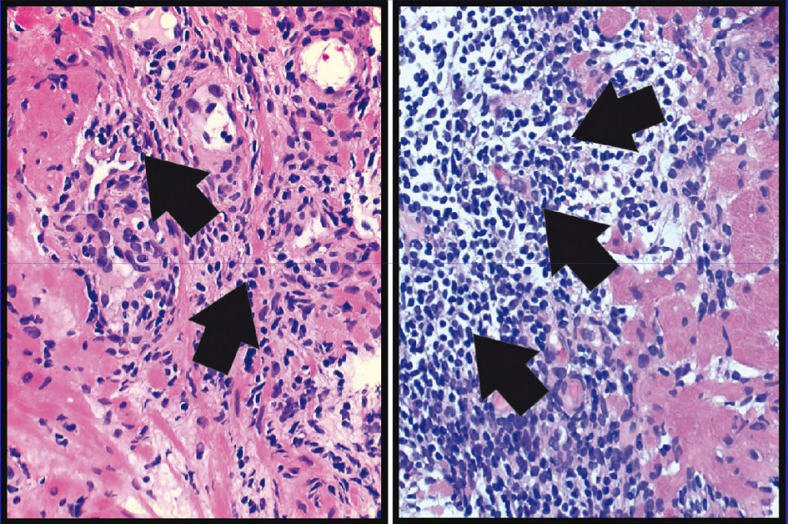

Mild chronic inflammation
Moderate chronic inflammation

Figure 1.

Of the $15.4 \%$ of men who had acute inflammation at baseline, $97.9 \%$ had mild, $1.9 \%$ had moderate, and $0.2 \%$ had severe (not shown in figure) inflammation. Of the $77.6 \%$ who had chronic inflammation, $89.0 \%$ had mild, $10.7 \%$ had moderate, and $0.3 \%$ had severe (not shown in figure) inflammation. 
Table 1

Baseline patient characteristics and International Prostate Symptom Score (IPSS) score and subscores by maximum chronic inflammation grade

\begin{tabular}{lcccc}
\hline Characteristic & Total & Maximum chronic inflammation & \\
& population & $\begin{array}{c}\text { Yes } \\
\text { (grade 1, 2, or 3) }\end{array}$ & $\begin{array}{c}\text { No } \\
\text { (grade 0) }\end{array}$ & p value \\
\hline Age, yr & & $62.9(6.0)$ & $62.3(6.2)$ & 0.0002 \\
PSA level, ng/ml & $62.8(6.1)$ & $5.9(2.0)$ & $6.0(2.0)$ & 0.0017 \\
Prostate volume, cc & $5.9(2.0)$ & $46.5(19.0)$ & $43.4(17.9)$ & $<0.0001$ \\
Average acute inflammation & $45.8(18.8)$ & $0.08(0.20)$ & $0.02(0.10)$ & $<0.0001$ \\
Average chronic inflammation & $0.06(0.18)$ & $0.61(0.38)$ & - & $<0.0001$ \\
Total IPSS score & $0.47(0.42)$ & $8.8(5.7)$ & $8.2(5.7)$ & $<.1(2.8)$ \\
Irritative subscore & $8.7(5.7)$ & $4.3(2.8)$ & $4.2(3.8)$ & $<0.0001$ \\
Obstructive subscore & $4.3(2.8)$ & $4.4(3.7)$ & $1.5(1.2)$ & 0.0013 \\
Nocturia subscore & $4.4(3.7)$ & $1.6(1.2)$ & 0.0001 \\
\hline
\end{tabular}

All values are mean $( \pm \mathrm{SD})$. 
Nickel et al.

Page 9

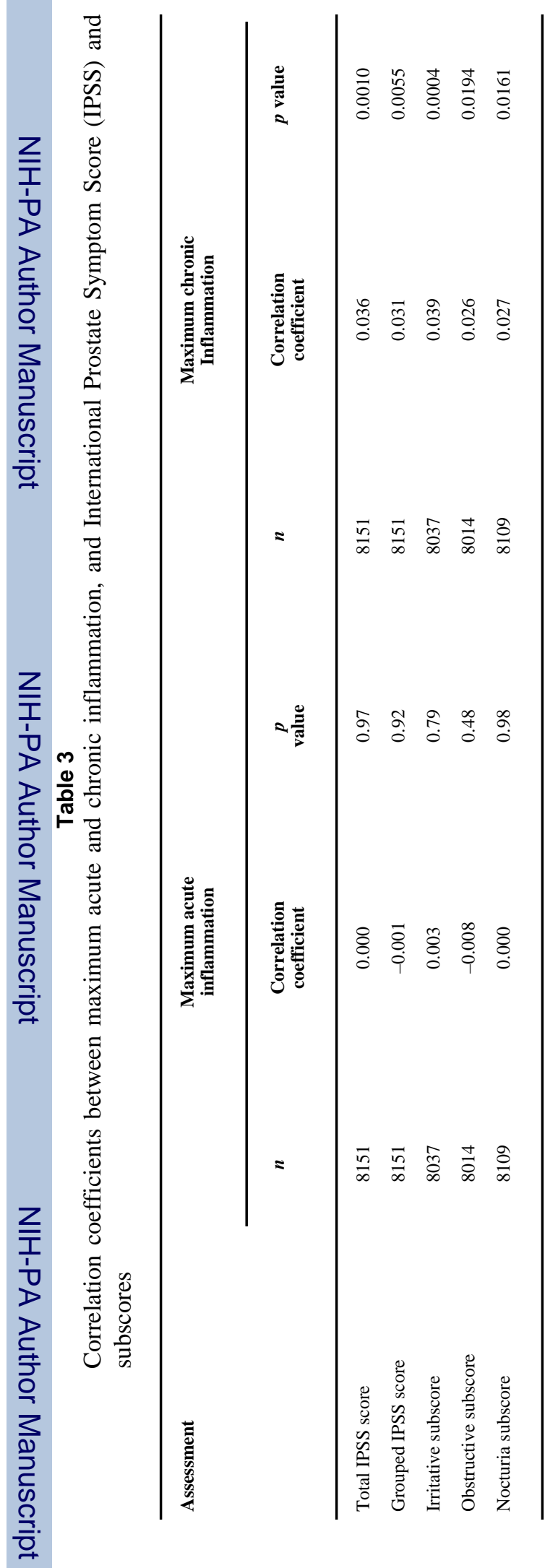

Eur Urol. Author manuscript; available in PMC 2009 December 1. 